\title{
Unidades didácticas como estrategia para fortalecer la competencia científica explicación de fenómenos
}

\author{
Didactic units as a strategy to strengthen scientific \\ competence explanation of phenomena
}

\begin{abstract}
Unidades didáticas como estratégia para fortalecer a explicação da competência científica dos fenômenos
\end{abstract}

\author{
Danny Javier Suárez Velandia \\ Ingeniero de producción biotecnológica \\ Universidad Francisco de Paula Santander \\ dajasuve@gmail.com
}

\section{Resumen}

La presente investigación se realizó con estudiantes de sexto grado de la Institución Educativa Nuestra Señora del Carmen en la asignatura de Biología. La propuesta nace del análisis de las pruebas SABER 2016 según ICFES (2016) de los grados $5^{0}$ y $9^{\circ}$, las cuales permitieron observar debilidades en el área de Ciencias Naturales. Estas debilidades fueron marcadas por bajos resultados en la competencia explicación de fenómenos, por lo cual se determinó utilizar unidades didácticas como la estrategia para fortalecer esta competencia.

Se utilizó una metodología de investigación acción con enfoque cualitativo. El desarrollo de la investigación inició con una prueba diagnóstica, que permitió determinar el estado inicial de los estudiantes para la competencia explicación de fenómenos.

Se implementaron dos unidades didácticas bajo la temática del funcionamiento celular. Cada una de estas unidades se desarrolló mediante guías impresas utilizando actividades de juego de roles, analogías, relaciones causa y efecto, modelación y actividades experimentales.

Como resultado de la estrategia implementada se logró fortalecer la modelación de los estudiantes, la relaciones causa y efecto y la motivación de los estudiantes. 
Se puede concluir que el uso de unidades didácticas permitió el fortalecimiento de la competencia explicación de fenómenos en los estudiantes de la institución educativa Nuestra Señora del Carmen.

Palabras claves: Unidad didáctica, competencia científica, explicación de fenómenos.

\begin{abstract}
The present investigation was carried out with students of the sixth grade of the Educational Institution of Our Lady of Carmen in the subject of Biology. The proposal was born from the analysis of the SABER 2016 tests according to ICFES (2016) of grades 5 and 9, which allowed observing weaknesses in the area of Natural Sciences. These weaknesses were marked by low results in the explanation of phenomena, so it was determined to use didactic units as the strategy to strengthen this competence.
\end{abstract}

An action research methodology with a qualitative approach was used. The development of the research began with a diagnostic test that allowed to determine the initial state of the students for the explanation of phenomena competence.

Two didactic units were implemented under the theme of cellular functioning. Each of these units was developed through printed guides using role play activities, analogies, cause and effect relationships, modeling and experimental activities.

As a result of the implemented strategy, it was possible to strengthen student modeling, cause and effect relationships and student motivation.

It can be concluded that the use of teaching units allowed for the strengthening of the competence explanation of phenomena in the students of the educational institution Nuestra Señora del Carmen.

Keywords: Didactic unit, scientific competence, explanation of phenomena

\title{
Resumo
}

A presente investigação foi realizada com alunos do sexto ano do Instituto de Ensino de Nossa Senhora do Carmo, na disciplina de Biologia. A proposta nasceu da análise dos testes SABRE 2016 de acordo com o ICFES (2016) das $5^{\mathrm{a}}$ e $9^{\mathrm{a}}$ séries, o que permitiu observar fragilidades na área de Ciências Naturais. Essas fragilidades foram marcadas por baixos resultados na explicação dos fenômenos, por isso, optou-se por utilizar as unidades didáticas como estratégia para fortalecer essa competência. 
Uma metodologia de pesquisa-ação com abordagem qualitativa foi utilizada. O desenvolvimento da pesquisa começou com um teste diagnóstico que permitiu determinar o estado inicial dos estudantes para a explicação da competência dos fenômenos.

Duas unidades didáticas foram implementadas sob o tema do funcionamento celular. Cada uma dessas unidades foi desenvolvida por meio de guias impressos, utilizando atividades de dramatização, analogias, relações de causa e efeito, modelagem e atividades experimentais.

Como resultado da estratégia implementada, foi possível fortalecer a modelagem dos alunos, as relações de causa e efeito e a motivação dos alunos.

Pode-se concluir que o uso de unidades de ensino possibilitou o fortalecimento da explicação da competência dos fenômenos nos discentes da instituição de ensino Nuestra Señora del Carmen.

Palavras-chave: Unidade didática, competência científica, explicação dos fenômenos.

Introducción

De acuerdo al plan de mejoramiento institucional, se realiza año tras año una revisión de los resultados de las pruebas SABER cómo punto de partida para la toma de decisiones que mejoren la calidad educativa, y así aumentar el Índice Sintético de Calidad Educativa (ISCE) y por ende la formación integral de los estudiantes.

En las áreas que evalúa el ICFES en las pruebas SABER, están las ciencias naturales y educación ambiental, para esta área Quintanilla (2010) señala:

La rápida evolución que sufre esta disciplina le genera un dinamismo tal que continuamente surgen dudas, problemas y preguntas de interés tanto científico como social, en este contexto, la enseñanza de la biología y la educación ambiental, requiere del uso de estrategias que faciliten la comprensión y el aprendizaje de conceptos contextualizados y basados en los modelos y teorías que le dieron origen. Es decir, aproximar cada vez más la interpretación de los fenómenos a los modelos que propone la comunidad científica (p. 5).

Después de analizar los bajos resultados obtenidos por los estudiantes de la Institución Educativa Nuestra Señora del Carmen en las pruebas SABER de los grados 5 y 9 en ciencias naturales, se observa la necesidad de buscar alternativas eficientes en los procesos pedagógicos que les permita a los estudiantes 
desarrollar la competencia científica explicación de fenómenos y poder superar los bajos niveles en esta área.

Este trabajo inicia con el planteamiento de la pregunta de investigación: ¿Cómo fortalecer la competencia científica explicación de fenómenos por medio del área de biología en los estudiantes de sexto grado de la institución educativa Nuestra Señora del Carmen del municipio de Salazar de las Palmas? De acuerdo a esta pregunta de investigación se planteó el siguiente objetivo general: Fortalecer la competencia científica explicación de fenómenos por medio del área de biología mediante la implementación de unidades didácticas en los estudiantes de sexto grado de la Institución Educativa Nuestra Señora del Carmen del municipio Salazar de las Palmas.

El proyecto propuso la creación de dos unidades didácticas, que de manera planificada y acorde con el modelo pedagógico activo que se implementa en la institución educativa, fortalezcan la competencia científica explicación de fenómenos y así lograr la formación integral de los estudiantes y mejorar los resultados de las pruebas externas e internas.

\section{Metodología}

El proceso metodológico de la investigación es de tipo cualitativo, con un enfoque de investigación acción, que según Kemmis (como se citó en Latorre 2003) lo define como:

Es una forma de indagación autoreflexiva realizada por quienes participan en las situaciones sociales para mejorar la racionalidad y la justicia: a) Sus propias prácticas sociales o educativas. b) Su comprensión sobre las mismas. c) Las situaciones e instituciones en que estas prácticas se realizan.

Durante el desarrollo del presente trabajo de investigación se siguieron los pasos establecidos por Kemmis (como se citó en Latorre 2003): Planificar, actuar, verificar $\mathrm{y}$ reflexionar. A continuación se detallará en qué consiste cada una de estas fases.

\section{Planificar}

Se revisaron los resultados de las pruebas saber y las debilidades en el área de ciencias naturales y se establecieron una serie de actividades enmarcadas en el diseño y aplicación de unidades didácticas en biología de grado sexto, teniendo como base los fenómenos a nivel celular. En esta fase se determinaron las actividades que promovieran en los estudiantes la competencia explicación de fenómenos.

\section{Actuar}

En esta fase se implementaron dos unidades didácticas, bajo la metodología de Jorba y Sanmartí (como se citó en Gallego 2014, p.4) 
donde se contemplan cuatro fases; la fase de exploración, fase de introducción de nuevos aprendizajes, fase de estructuración y síntesis y fase de aplicación. Antes de iniciar cada fase se realizó una prueba diagnóstica para determinar el nivel de la competencia explicación de fenómenos.

La primera unidad didáctica, trato la temática de la célula y la funcionalidad de sus componentes. Se desarrolló mediante la aplicación de siete guías impresas, distribuidas en cada una de las fases propuestas por la metodología de Jorba y Sanmartí (como se citó en Gallego 2014. p.4).

La segunda unidad didáctica se encargó de generar aprendizajes sobre los fenómenos que ocurren en la membrana celular, al igual que la primera unidad didáctica, en esta se implementaron siete guías impresas.

Al finalizar las dos unidades y de acuerdo a lo establecido en el Proyecto Educativo Institucional (PEI), se realizó una prueba final con preguntas tipo SABER, la cual arrojó muy buenos resultados. Ya qué, la mayoría de estudiantes obtuvieron calificaciones en un nivel básico y alto.

\section{Observar}

Esta fase se realizó de manera paralela a la fase de implementación, para esto se utilizó el diario de campo, donde se registraron las diferentes situaciones producto de la aplicación de las unidades didácticas en la población de estudiantes de grado sexto. Cabe aclarar que para esta investigación al existir un solo grado sexto, este será también la muestra.

\section{Reflexionar}

$\mathrm{Al}$ igual que la fase anterior, ésta fase se realizó de manera paralela a la observación, y al finalizar cada una de las actividades de las unidades didácticas, para realizar los ajustes producto de la realidad observada.

Para establecer la población que participo en esta investigación tomaremos el concepto de Bernal (2010) quien dice. "La población es la totalidad o el conjunto de todos los sujetos o elementos que tienen ciertas características similares y a los cuales se refiere la investigación" (p.189). De la misma forma este autor dice "la muestra es la parte de la población que se selecciona de la cual realmente se toma la información" (p.189). Así, para el desarrollo de este proyecto de investigación se tomó como población a los 36 estudiantes de grado sexto de la Institución educativa Nuestra Señora del Carmen. Para esta investigación, la muestra y la población son las mismas, ya que existe un solo grado sexto. Se solicitó el consentimiento informado a los padres de familia.

Se establecieron dos categorías como criterio para el análisis de los 
resultados observados. La primera categoría correspondía a la competencia explicación de fenómenos dentro de la cual se analizaron las siguientes subcategorías: Busco o formulo razones a fenómenos o problemas, creo argumentos lógicos y propositivos de los fenómenos percibidos, explico un mismo fenómeno utilizando representaciones conceptuales pertinentes a diferentes grados de complejidad, establezco relaciones de causa efecto, identifico y uso modelos biológicos para explicar fenómenos de la naturaleza. También se definieron subcategorías para la metodología, con las que se analizaron los recursos, el ambiente de aprendizaje y las actividades realizadas.

\section{Resultados}

Los resultados de esta investigación se presentan como producto de las observaciones registradas en el diario de campo y el análisis de actividades que los estudiantes desarrollaron mediante las unidades didácticas, teniendo en cuenta los referentes teóricos. De acuerdo a lo anterior se contrastaron las categorías y subcategorías establecidas y los hallazgos encontrados durante la implementación de las mismas.

De acuerdo a los objetivos establecidos se realizaron pruebas diagnósticas para cada unidad didáctica, con el fin de determinar el estado inicial de los estudiantes para la competencia explicación de fenómenos. Estas pruebas fueron diseñadas bajo preguntas tipo ICFES, específicas para esta competencia.

Como resultado de esta actividad en cada una de las pruebas diagnósticas, se observó un bajo nivel en la competencia explicación de fenómenos en la mayoría de los estudiantes.

A nivel general un factor que llamó la atención fue la búsqueda de aprobación de la mayoría de estudiantes durante las actividades realizadas, mostrando una conducta de dependencia con el docente. De igual forma se observó poca motivación cuando las actividades involucraban lecturas, esto al igual que lo nombrado anteriormente revelaron dificultades y desinterés en el momento de leer.

Uno de los resultados más importantes que se pudo observar durante el proceso de investigación, fue un progreso constante en cuanto a la identificación y uso de modelos para explicar los diferentes fenómenos estudiados, teniendo en cuenta que a la mayoría de estudiantes habían tenido un acercamiento de la temática en quinto grado.

También es de resaltar que las actividades propuestas causaron un efecto positivo en la capacidad que tienen los estudiantes para relacionar causa efecto, factor relevante para la explicación de fenómenos. Así mismo, es 
importante decir que unas de las falencias evidenciadas durante el proceso fueron el bajo nivel de lectura y el desconocimiento de conceptos básicos para la temática estudiada.

Para finalizar se realizó una prueba tipo ICFES, que en buena parte de los estudiantes de grado sexto arrojó buenos resultados con calificaciones de nivel Básico y algunos en nivel superior. Lo anterior reafirma que, la intervención con las unidades didácticas causo impactos positivos en la competencia explicación de fenómenos.

Hay que destacar también que la realización de las actividades como el juego de roles y las que implicaron construcción de modelos, causaron un impacto positivo para el desarrollo de la competencia explicación de fenómenos, ya que los estudiantes modelaron la célula y la membrana celular y reflexionaron al final del proceso. De esa manera, coincide con lo dicho por Ziman (como se citó en Blanco 2016) "las observaciones del mundo natural se realizan a través de un modelo que puede ser establecido por la comunidad científica o puede ser el modelo propio del individuo" (p.506). Estas actividades mostraron el progreso de los estudiantes, ya que cumplían con una de las subcategorías establecidas en esta investigación como lo fue la modelación. De estas actividades también se debe rescatar que se presentó un impacto inesperado en otros grados de la institución, quienes manifestaron su interés por tener ese tipo de actividades en sus clases. Lo cual permite reflexionar y deja un punto de partida para que sean incluidas en el plan de asignatura de los otros grados.

\section{Conclusiones}

Después de finalizar este trabajo de investigación y respondiendo a la pregunta de investigación se puede decir que las unidades didácticas aplicadas teniendo en cuenta actividades de modelación, experimentación y relaciones de causa y efecto, fortalecieron la competencia científica explicación de fenómenos en los estudiantes de grado sexto de la institución Educativa Nuestra Señora del Carmen.

La aplicación de unidades didácticas tuvo un impacto positivo sobre la comunidad educativa, esto se evidenció a nivel motivacional, ya que los estudiantes trabajaron con entusiasmo y creatividad.

De igual forma, el impacto sobre la competencia científica explicación de fenómenos se evidencio durante todo el proceso, en las construcciones, modelaciones y explicaciones generadas por los estudiantes y mediante la aplicación de la prueba final en la cual se obtuvieron buenos resultados.

En relación con el primer objetivo que fue diagnosticar los niveles y oportunidades de mejora 
en la competencia científica explicación de fenómenos en biología en los estudiantes de sexto grado, se logró detectar gracias a la aplicación de la prueba diagnóstica un bajo nivel en la competencia explicación de fenómenos, un bajo nivel de lectura, dificultades en el reconocimiento de conceptos y poca motivación por la lectura.

Los resultados de este diagnóstico fueron importantes ya que sirvieron como punto de partida para diseñar estrategias que permitieran el fortalecimiento conceptual.

Con respecto al segundo objetivo que fue el diseño y la implementación de unidades didácticas, se logró evidenciar que la estructura de esta estrategia fue apropiada, ya que permitió que en los estudiantes se fortalecieran progresivamente la conceptualización, la modelación y la capacidad de realizar relaciones de causa y efecto en los fenómenos celulares.

De igual forma, se observó que el uso de la experimentación fue muy motivante e importante porque generó el contraste necesario para argumentar los fenómenos a nivel celular.

Otro aspecto importante que se debe destacar fue el bajo porcentaje de uso de la clase magistral, lo cual mejoró el ambiente de aprendizaje y permitió el descubrimiento y la construcción de los aprendizajes por parte de los estudiantes.

Con respecto a las debilidades observadas, se resalta el desinterés por la lectura, bajo nivel de lectura literal e inferencial.

En cuanto al último objetivo que fue analizar los alcances de las unidades didácticas sobre la competencia explicación de fenómenos, se puede decir que hubo un avance y un impacto positivo con la utilización de las unidades didácticas, esto se evidenció con el mejoramiento progresivo de la argumentación, modelación y explicación de los fenómenos a nivel celular por parte de la mayoría de los estudiantes.

\section{Referencias}

Bernal, C (2010). Metodología de la Investigación. Editorial PEARSON. Colombia.

Blanco, P (2017). Análisis del nivel de desempeño para la explicación de fenómenos de forma científica en una actividad de modelación. Disponible en https://revistas.uca.es/index.p hp/eureka/article/viewFile/31 58/3192 Fecha de consulta: 23 de Octubre de 2017.

Instituto Colombiano para el Fomento de la educación Superior (2017). Resultados pruebas SABER 3,5 Y 9. Documento en línea. Disponible en http://www2.icfesinteractivo.g 
ov.co/ReportesSaber359/cons

ultaReporteEstablecimiento.jsp

x. Fecha de consulta: 20 de

Octubre de 2017.

Gallego, D (2014). Unidades didácticas: Un camino para la transformación de la enseñanza de las ciencias desde un enfoque investigativo. Memorias, sexto congreso Internacional sobre formación de profesores de ciencias. Bogotá. Disponible en revistas.pedagogica.edu.co/ind ex.php/TED/article/download/ 3460/3055. Fecha de consulta: 17 de Noviembre de 2017.

Latorre, A (2003). La investigación acción: conocer y cambiar la práctica educativa. Barcelona: Grao. Disponible en https://www.uv.mx/rmipe/file s/2016/08/La-investigacionaccion-Conocer-y-cambiar-lapractica-educativa.pdf. Fecha de consulta: 17 de Noviembre de 2017.

Quintanilla, M (2010). Unidades didácticas en biología y educación Ambiental. Colombia. Disponible en: http://www7.uc.cl/sw_educ/e ducacion/grecia/plano/html/p dfs/destacados/LibroDBioGrec ia.pdf. Fecha de consulta: Fecha de consulta: 17 de Noviembre de 2017. 


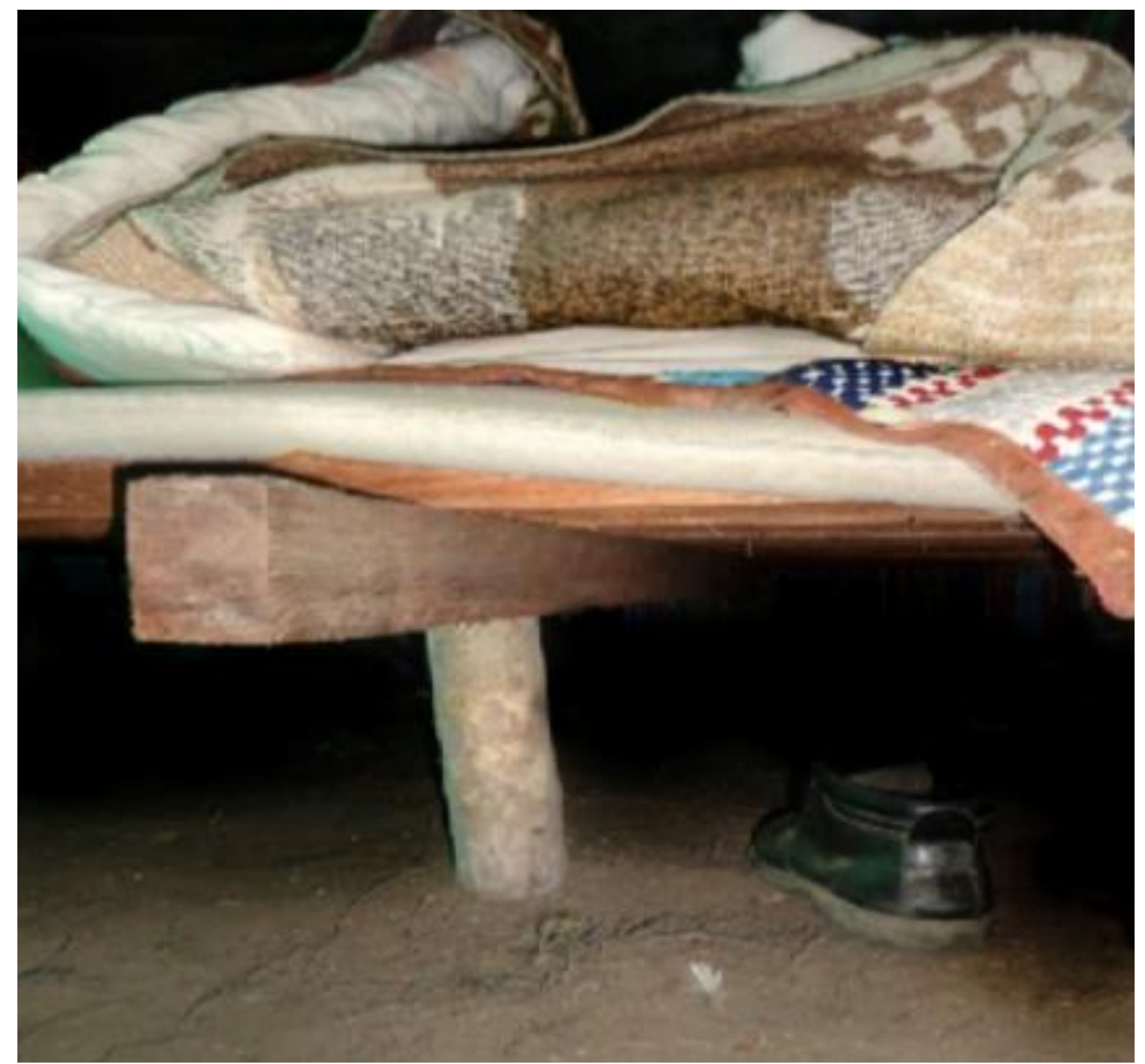

Número de la obra: 2

Título: "sobre camas"

Autor: Yenny Banessa Bonilla

Lugar: Zona rural municipio de Baraya

Finca: Bella Vista

Fecha de captura: Febrero 7 de 2014

Técnica: Fotografía digital

Fuente: Bonilla, Y. B. (2014). Sobre camas. Una mirada estética popular del municipio de Baraya. Tesis de pregrado. Universidad Surcolombiana. Neiva, 2014. p. 71. 